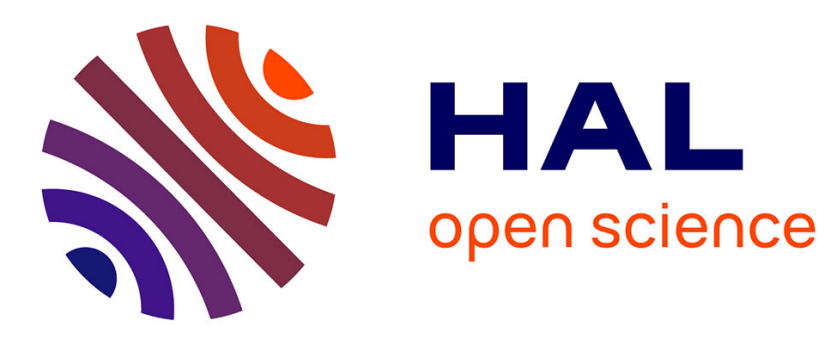

\title{
Capacitive Sensing for Improving Contact Rendering with Tangible Objects in VR
}

Xavier de Tinguy, Claudio Pacchierotti, Anatole Lécuyer, Maud Marchal

\section{To cite this version:}

Xavier de Tinguy, Claudio Pacchierotti, Anatole Lécuyer, Maud Marchal. Capacitive Sensing for Improving Contact Rendering with Tangible Objects in VR. IEEE Transactions on Visualization and Computer Graphics, 2021, 27 (4), pp.2481-2487. 10.1109/TVCG.2020.3047689 hal-03092809

\section{HAL Id: hal-03092809 \\ https://hal.inria.fr/hal-03092809}

Submitted on 2 Jan 2021

HAL is a multi-disciplinary open access archive for the deposit and dissemination of scientific research documents, whether they are published or not. The documents may come from teaching and research institutions in France or abroad, or from public or private research centers.
L'archive ouverte pluridisciplinaire HAL, est destinée au dépôt et à la diffusion de documents scientifiques de niveau recherche, publiés ou non, émanant des établissements d'enseignement et de recherche français ou étrangers, des laboratoires publics ou privés. 


\title{
Capacitive Sensing for Improving Contact Rendering with Tangible Objects in VR
}

\author{
Xavier de Tinguy, Student Member, IEEE, Claudio Pacchierotti, Senior Member, IEEE, \\ Anatole Lécuyer, Member, IEEE, and Maud Marchal, Member, IEEE
}

\begin{abstract}
We combine tracking information from a tangible object instrumented with capacitive sensors and an optical tracking system, to improve contact rendering when interacting with tangibles in VR. A human-subject study shows that combining capacitive sensing with optical tracking significantly improves the visuohaptic synchronization and immersion of the VR experience.
\end{abstract}

Index Terms - Haptic rendering, Human computer interaction, Sensors, Human-centered computing.

\section{INTRODUCTION}

$\mathrm{I}$ MMERSIVE Virtual Reality (VR) must actively engage one's senses, so as to make the user feel truly part of the virtual world. One important aspect to achieve this objective remains the synchronization of motion and sensory feedback between the human users and their virtual avatars. Whenever one user moves a limb, the same motion should be replicated by the avatar; similarly, whenever the avatar touches a virtual object, the user should feel the same haptic experience. Di Luca et al. [1] recently studied the range of tolerable visuohaptic asynchronies when touching an object. Participants could not reliably detect the asynchrony if haptic feedback was presented less than $50 \mathrm{~ms}$ after the view of the contact. The asynchrony tolerated for presenting haptic feedback before the visual one was instead only $15 \mathrm{~ms}$. These results suggest rather stringent requirements for haptic-enabled VR systems. Achieving this visuohaptic synchronization is also important for the perception of the objects properties. For example, Di Luca et al. [2] showed that delay in presenting visual and force information introduces a bias in the perception of compliance. Knorlein et al. [3] proved that a similar effect holds also when interacting with virtual objects.

To ensure a good match between the motion of the users with respect to their avatars, commercial VR systems already provide vision-based solutions able to track the headset or a dedicated active prop (e.g., the HTC Vive tracker). Other more advanced approaches consist in tracking a set of markers constellations worn directly by the user (e.g., Vicon and Optitrack systems). However, they require a clear line of sight and their performance significantly degrades in the presence of, e.g., occlusions, calibration and modeling errors, suboptimal light conditions or positioning of the markers. This limitation leads to mismatches in the relative positioning of the virtual hand with respect to the virtual object, i.e., a negative or positive virtual gap upon contact, breaking the synchronicity of the virtual and tangible contacts.

This paper proposes an innovative approach to tracking

- X. de Tinguy and M. Marchal are with Univ Rennes, INSA, Inria, CNRS, IRISA - Rennes, France. E-mail: xavier.de-tinguy@inria.fr, maud.marchal@irisa.fr.

- C. Pacchierotti is with CNRS, Univ Rennes, Inria, IRISA - Rennes, France. E-mail: claudio.pacchierotti@irisa.fr.

- A. Lécuyer is with Inria, Univ Rennes, CNRS, IRISA - Rennes, France. E-mail: anatole.lecuyer@inria.fr.

- This project has received funding from the European Union's Horizon 2020 programme under grant agreement No 801413; project "H-Reality". and rendering of contacts with tangible objects in VR, compensating such relative positioning error to achieve a better visuohaptic synchronization upon contact and preserve immersion during interaction in VR. We employ one tangible object to provide distributed haptic sensations. It is equipped with capacitive sensors to estimate the proximity of the user's fingertips to its surface. This information is then used to retarget, prior contact, the fingertips position as obtained from a standard vision tracking system, so as to achieve better synchronization between virtual and tangible contacts. The main contributions of our work can be summarized as follows:

- we propose a novel approach for enhancing contact rendering in VR when using tangible objects, instrumenting the latter with capacitive sensors;

- we design and showcase a sensing system and visuohaptic interaction technique enabling high contact synchronization between what users see and feel;

- we conduct a user study showing the capability of our combined approach vs. two stand-alone state-ofthe-art tracking systems (Vicon and HTC Vive) in improving the VR experience.

\section{Related Work}

Tangible objects. Using tangible props to mediate virtual interaction is common in VR, as it provides an easy and natural way to convey compelling shape and weight sensations [4], [5]. However, to render complex virtual environments, one needs to develop multiple ad-hoc tangible objects, which requires a lot of work, might be expensive, and even unfeasible in the case of shape-changing virtual environments. To address this issue, researchers have focused on techniques able to render multiple virtual objects through few tangible ones [6], [7], [8]. For example, Han et al. [6] used one tangible prop to render multiple virtual objects by re-targeting the reaching movements of users at runtime. More recently, de Tinguy et al. [7] matched the shape and weight of a tangible object to multiple virtual ones. Users were guided to grasp the virtual and tangible objects where their local haptic properties showed the most similarities.

Most works using tangible props in VR track the movements of the human user via external optical trackers, such as the Vicon or the Optitrack, limiting occlusions by simply employing few tangible objects. 


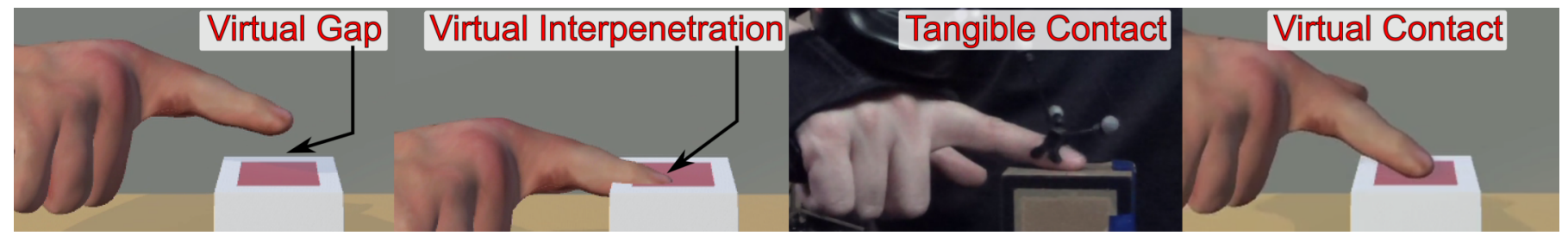

Fig. 1. Representative issues while using standard optical tracking systems (left) vs. our integrated capacitive-based approach (right). By combining tracking information from standard optical tracking systems with proximity information from a capacitive sensor, we are able to re-target the virtual fingertip toward the virtual surface, achieving a better synchronization between tangible and virtual contacts.

Capacitive sensing. As the human body is naturally behaving like a capacity, capacitive sensing can be used to measure the distance or detect a contact between the sensor and the human skin [9], [10]. For example, Hinckley et al. [9] used capacitive sensing to improve user experience when interacting with a mouse or a trackball. Sato et al. [10] monitored the response to capacitive human touch over a range of frequencies and were able to discriminate between individuals and the nature of the interaction with the sensor. Using a grid of capacitive electrodes makes possible to track a user's finger on a plane, which is nowadays how we interact with most tactile/touch screens [11]. Yu et al. [12] proposed a similar approach to track tangible objects on a capacitive screen. They used active modulation circuits embedded in the tangible objects to encode additional IDs information and reduce the contact area of the spatial tags. Even further, Rekimoto et al. [13] developed a sensor architecture for making interactive surfaces sensitive to hand and finger gestures. It was constructed by laying a mesh of transmitter/receiver capacitive electrodes on the surface. Similar capacitive-based grids and artificial skins have often been used in robotics to endow manipulators and humanoids with tactile sensing capabilities [14], [15]. MacRitchie and McPherson [16] combined capacitive sensing (contact-only) and high-speed camera tracking to sense both the small-scale motion of fingers and the large-scale movement of the hands and arms during piano playing.

Although capacitive sensing is a widespread technology, to the best of our knowledge, it has not been used before for attaining synchronicity of interaction between a tangible object and its virtual counterpart.

Contact rendering in VR. Several works focused on the transition between making and breaking contact [17], [18], [19], [20], [21]. For example, Vechec et al. [19] opted for a wearable haptic device made of several actuators which can be controlled to engage or disengage a pin against the user's skin, at high rate, using electromagnetic actuators. For example, Kuchenbecker et al. [20] endowed a grounded haptic device with a passive cutaneous end-effector. The kinesthetic feedback provided by the grounded interface bends the internal springs of the display and brings a shell in contact with the user's finger, providing the sensation of making/breaking contact with the rendered surface. To attain visuohaptic synchronicity, a promising technique is to redirect contacts with a virtual object toward the corresponding tangible one. This can be done by exploiting the dominance of vision over the other senses. For example, Kohli [22] warped the space around a virtual object to redirect the motion of the user fingertip, so as to synchronize the contact between the tangible prop and the virtual environment. Similarly, to highlight the moment of contact, Sreng et al. [23] proposed to signal the proximity and force of the contact with changing colored glyphs. More recently, Cheng et al. [24] simulated interactions with the virtual scene's geometry by redirecting the user's hand to a matching primitive on the tangible environment. Of course, being able to robustly tracking the fingers and hand position is of paramount importance. External optical trackers, such as the Leap Motion, Optitrack, and Vicon, are still the go-to solutions for most hapticenabled VR applications [25], [26]. More recently, non-optical approaches have also gained some popularity, using IMU[27] or magnet-based [28] techniques.

For the first time, we combine retargeting motion techniques with the tracking coming from global optical trackers and local capacitive sensing, as to achieve high visuohaptic synchronicity of contact. Our approach is compatible with most global tracking systems as long as the virtual hand can be retargeted toward the surface of the virtual object.

\section{Method}

A visuohaptic interaction technique combines the information from the instrumented tangible object with that from an external vision tracking system, aiming at a better visuohaptic synchronization during contact interaction in VR. Indeed, as the user approaches and then touches the tangible object, the virtual environment should render as closely as possible the approach and contact of the hand avatar with the corresponding virtual object. As to compensate for mismatches and positioning errors, we propose to retarget the motion of the virtual hand along the normal of the objects surface. To do so, we combine the local estimation of the signed distance along this direction from the capacitive sensor with the global tracking information coming from a commercially-available optical tracker. In the following section, distance will always refer to the projected component along the normal direction to the targeted surface, i.e., the height in the surface local frame.

\subsection{Apparatus}

The experimental setup illustrating the principle of our approach is shown in Fig. 2. It is composed of a tangible cube, instrumented on three of its faces with 2-cm-wide copper electrodes, acting as our proximity sensors. The cube is fixed on a rigid structure placed on a table in front of the human user, so as to guarantee precise and reproducible settings. The user wears an HTC Vive head-mounted display (HMD) on the head, a Vive Tracker on the back of the hand, and an attachment with three reflective markers on the back of intermediate phalanx of the right index finger. The Vive 


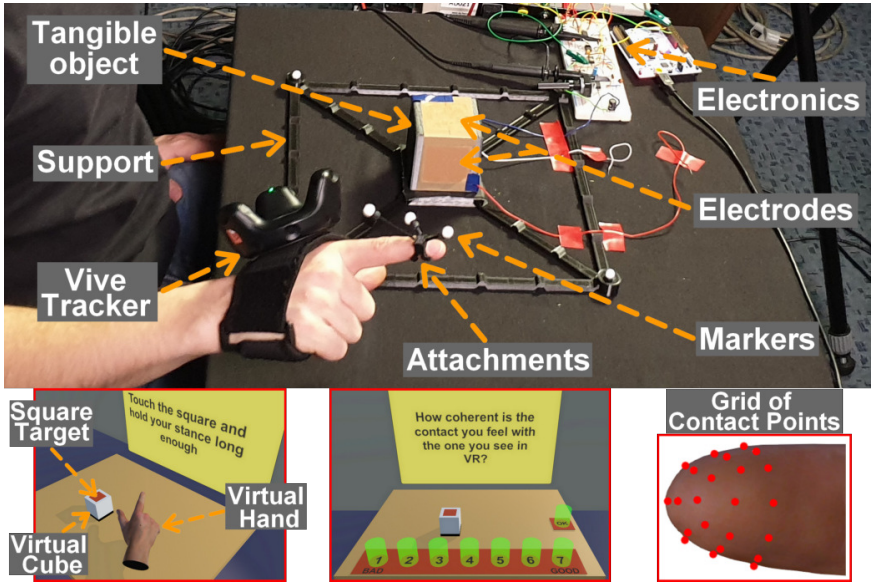

Fig. 2. Apparatus. Insets show the virtual environment, the display panel, and the grid of virtual contact points.

Tracker enables the use of the HTC Vive Lighthouse optical tracking system, while the reflective markers enable the use of the Vicon Bonita optical tracking system. The two optical tracking systems are never used at the same time. They can either be used alone or in combination with the capacitive sensing system (see Sec. 4). The fingertip is always left free to interact with the tangible object.

\subsection{Capacitive Sensing}

As said above, a tangible cube is instrumented with capacitive sensor made of copper layers on three of its faces, so as to provide information on the distance between the object and the user's hand. The capacity sensed increases as the user's hand gets close to the tangible surface. The detection circuit is composed of an RLC oscillator with an additional copper electrode connected between $L$ and $C_{0}$, thus acting as a second capacitor $C_{p}$ (see Fig. 3).

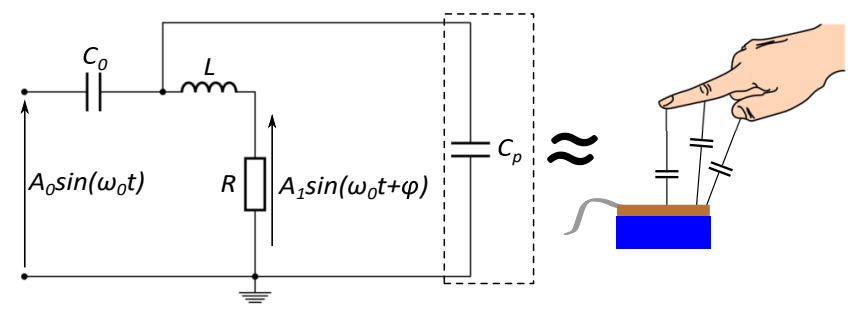

Fig. 3. RLC Oscillator sensible to the variation of the capacitor $C_{p}$.

The resonance frequency of this oscillator being $f=$ $1 /\left(2 \pi \sqrt{L\left(C_{0}+C_{p}\right)}\right)$, the excitation frequency will always be $f_{0}$, set as the resting resonance frequency, i.e., the resonance frequency at which the user is infinitely far from the electrode. Whenever the user's hand is getting close to the electrode, as $C_{p}$ increases, the resonance frequency decreases and drifts away from $f_{0}$, resulting in the output's phase $\varphi$ to shift and the amplitude $A_{1}$ to decrease. By filtering the multiplication of the input $A_{0} \sin \left(\omega_{0} t\right)$ and output $A_{1} \sin \left(\omega_{0} t+\varphi\right)$ of the oscillator (Fig. 3), we obtain a continuous component $s \propto$ $A_{1} \cos (\varphi)$, which is an estimation of the user proximity.

\subsection{Signal characterization}

The relationship between the capacitive signal and the user's proximity is dependent on many factors, e.g., the users' hands morphology and pose, the electrode's size. The signal is not always fully consistent and accurate, and will have small variations around a mean behavior which we will fit as to serve as a reference for the visuohaptic retargeting.

Five participants were asked to close their right hand in a fist with the index finger pointing out (as in Fig. 3) and then move steadily along a straight line normal to the electrodes, as displayed through the HMD. They carried out 6 back-and-forth movements of at least $40 \mathrm{~cm}$. Using these data, we fitted a mean curve between the signal coming from the capacity sensor vs. the Vicon-measured distance from the electrode registered using the Vicon Bonita system. We used the nlinfit MATLAB tool to fit this curve:

$f_{f i t}(z)=\left(\frac{a_{1}+1}{\left(1+\exp \left(-a_{2}\left(z+a_{3}\right)\right)^{\frac{1}{a_{4}}}\right.}-1\right)\left(1+\exp \left(-a_{5} z\right)^{a_{6}}\right)$

Fig. 4a shows the acquired points as well as the fitted curve, and highlight a common behavior across participants, with variations around the mean fitted curve.

The procedure was repeated for each of the three electrodes, so as to evaluate a mean behavior for each of them and prepare lookup tables for estimating the distance from the capacitive signal (see Sec. 3.2).

\subsection{Visuohaptic retargeting and contact rendering}

Now that we know how to relate the data gathered from the capacitive sensing with the user's proximity to the tangible object, we aim at combining it with the the optical tracking system by performing an interpolation between the two along the surface's normal.

Let us call $z_{\text {capa }}$ the distance between the user and the tangible object as estimated by the capacitive system (see Sec. 3.3) and $\alpha$ its normalized value, i.e., $z_{\text {capa }}=\alpha z_{M}$ with $z_{M}$ being the maximum detection range (e.g., $z_{M}=40 \mathrm{~cm}$ for our top electrode). Similarly, let us call $z_{t r a c}$ the distance between the user and the tangible object as estimated by the optical tracking system. As seen on Fig. $4 \mathrm{~b}$, when the user is far from the electrode, $\alpha$ (and thus $z_{\text {capa }}$ ) is too noisy to rely on it. In this situation, we should mostly rely on the external tracking system. On the other hand, when the user is close to the electrode, the external tracking system might suffer from occlusions and other limiting factors (see Sec. 1), while the capacitive estimation is precise and reaches 0 upon contact. In this situation, we should mostly rely on the capacitive sensing. To do so, we define a transformed version of $\alpha$, which spans between 0 and 1 in the range $\left[\alpha_{\mathrm{m}}, \alpha_{\mathrm{M}}\right]$, i.e.,

$$
\alpha_{t h}= \begin{cases}0 & \text { if } \alpha \leq \alpha_{\mathrm{m}} \\ \frac{\alpha-\alpha_{m}}{\alpha_{M}-\alpha_{m}} & \text { if } \alpha_{\mathrm{m}}<\alpha<\alpha_{\mathrm{M}}, \\ 1 & \text { if } \alpha \geq \alpha_{\mathrm{M}}\end{cases}
$$

Fig. 4b shows $\alpha$ and $\alpha_{t h}$ for one representative user during six back-and-forth movements. Finally, we can proceed with retargeting the position of the user's finger according to both tracking information, i.e.,

$$
z_{\text {fin }}=\alpha_{t h} z_{\text {trac }}+\left(1-\alpha_{t h}\right) z_{\text {capa }} .
$$

Whenever the user is farther than $\alpha_{\mathrm{M}}$ from the electrode, we only consider information coming from the optical tracking system, disregarding the capacitive sensing data. When the user is between $\alpha_{\mathrm{M}}$ and $\alpha_{\mathrm{m}}$, we consider a combination of 

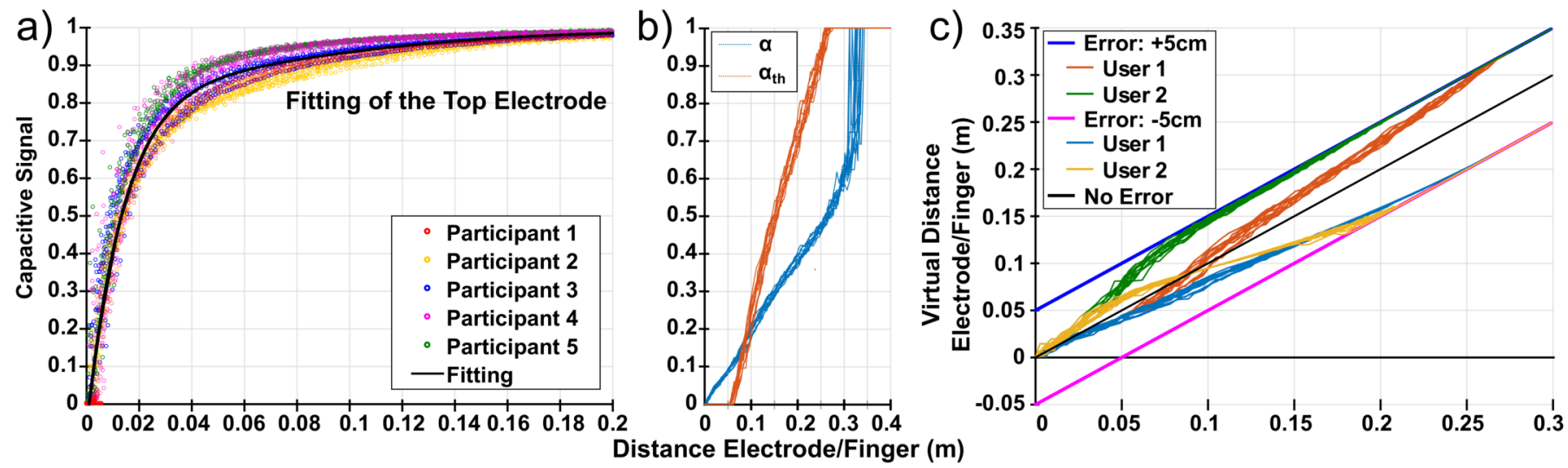

Fig. 4. Visuohaptic retargeting. a) Acquired signal from the top electrode vs. Vicon-measured distance from it. The best fit is plotted as a solid black line. The capacitive signal ranges from 1 to 0 with respect to the user's proximity, from the edge of the detection range (1) to the contact (0). $\mathrm{b}) \alpha$ and $\alpha_{t h}$ processed from a participant's data. c) Example: The optical tracking system measures the user-object distance with an error of $+5 \mathrm{~cm}$ (blue solid line) or $-5 \mathrm{~cm}$ (pink solid line), while the capacitive sensing system has no estimation error. Using only optical tracking, the virtual and tangible contacts are of course not synchronized. Combining proximity capacitive data and optical tracking mitigates this mismatch. Plots of User 1 and User 2 show this behavior (orange and green for the case with an error of $+5 \mathrm{~cm}$; cyan and yellow for the other): despite the error of the optical tracking system, the combined estimated position is corrected as the user-object distance diminishes.

the two, starting to smoothly include capacitive data at $\alpha_{\mathrm{M}}$ and weighting it more and more as we approach $\alpha_{\mathrm{m}}$. When the user is closer than $\alpha_{\mathrm{m}}$ to the tangible object, we only consider information coming from the capacitive sensing system, disregarding the optical tracking data.

Fig. 4c shows four representative examples of this approach. We consider two situations where the optical tracking system measures the distance between the user and the tangible object with an error of either $+5 \mathrm{~cm}$ (blue solid line) or $-5 \mathrm{~cm}$ (pink solid line), while the capacitive sensing system works correctly (no estimation error). The retargeting was computed by adding those simulated error to the trajectory acquired for two of the participants during the signal characterization of Sec. 3.3. If no capacitive sensing is employed, users either touch the tangible object before the contact in the virtual environment happens or touch the virtual object before the contact with the tangible object happens. Either way, this is expected to degrade the resulting interaction. Combining the proximity capacitive data with the optical one enables us to address this mismatch, improving the quality of the contact rendering. The green and red lines of Fig. 4c show how the combined estimated position $z_{f i n}$ is corrected as the distance between the user and the tangible object diminishes, leading to an increased visuohaptic synchronization and higher immersion at the contact.

Of course, it is important to highlight that capacitive sensing only provides information regarding the proximity of the user's finger to the electrode, conveying no information on the orientation of the finger itself. This information comes only from the global tracking system. For this reason, while the position of the virtual avatar is adjusted according to the visuohaptic retargeting mechanism describe above, its orientation with respect to the tangible object is not. We consider the finger to be oriented as estimated by the global optical tracking system and the distance detected by the capacitive sensor to be that between the tangible object and the closest point on the finger. Thus, we apply the retargeting to the closest point of the virtual finger to the virtual object's surface. To be able to carry out this adjustment at runtime, we consider a grid of 23 points spread around the virtual fingertip (see Fig. 2-left inset). These points are placed below the virtual skin surface to allow interpenetration as if some deformation of the virtual finger would occur upon contact.

\section{USER STUdY}

We carried out a user study to assess the performance and viability of our combined capacitive-optical contact rendering approach vs. standard optical-only tracking techniques when interacting with tangible objects in VR. The three hypotheses of our study are:

- H1: Using our capacitive sensing system together with an optical tracking system improves the perceived coherency and synchronicity of the contact.

- H2: H1 holds both for brief contacts (e.g., tapping on the tangible object) and sustained contacts (e.g., apply a constant pressure on the tangible objects).

- H3: Seeing the virtual distance between the fingertip and the object (lateral point of view) increases the perception of any existing discrepancy vs. having an occlusion between the fingertip and the object (contact with back or top faces of the cube, see Fig. 2).

\subsection{Experimental Methods}

\subsubsection{Participants}

18 participants ( 12 males, 6 females, age $\mathrm{M}=28.5$ and $\mathrm{SD}=$ 0.88 , right-handed) took part in the experiment.

\subsubsection{Experimental setup}

We used the setup described in Sec. 3.1 and shown in Fig. 2, equipping the participant with both a Vive Tracker and three reflective markers for the Vicon. The tangible object was instrumented with three electrodes, on the top, right, and back faces of the cube. As the Vive Tracker was placed on the back of the hand, we asked the participants to always keep their hand closed in a fist, with the index finger pointing out (as in Figs. 2 and 3). The virtual environment was composed of a virtual version of the tangible object, which position was carefully set. The object's face the participant had to interact with was colored in red. To help participants assess their distance from the tangible object, we cast the shadow of the virtual finger onto the object using the standard Unity3D rendered. 


\subsubsection{Experimental procedure}

Participants performed two interaction tasks: "touching", where they had to approach the target object's face and touch it for 3 seconds without breaking contact; and "tapping", where they had to approach the object's face and tap on it 10 times. Participants were asked to contact the object naturally, as they would do with a real object. For the tapping task, they were encouraged to explore different contact orientations/angles at each tap. At the end of each task repetition, participants answered the question (Q0) "How coherent is the contact you felt with the one you saw in VR?" (see lower inset of Fig. 2) using a 7-item Likert-like scale.

The experiment started with a written explanation of the procedure and the signature of a consent form. Then, participants watched a short video demonstrating the two tasks. Finally, we accurately calibrated the tracking system(s) in use for the considered session. Three practice trials per task were allowed to ensure that the participant had well understood the instructions. At the end of the experiment, participants filled a final subjective questionnaire.

\subsubsection{Experimental Design}

We used two different optical tracking systems. The Vicon system is known to be precise and reliable and it was considered as the reference or state-of-the-art condition. The HTC Vive tracker is one of the most affordable VR solutions, but its usage comes with some drawbacks: more jitters, drifts, and higher position and orientation errors with respect to Vicon-based frameworks. Moreover, it requires a tracking device which is more cumbersome than the reflective markers used by the Vicon. For this reason, the Vive tracking element was worn on the back of the hand while the Vicon's reflective markers were placed on the back of the intermediate phalanx of the right index finger (see Fig. 2). As we aim at tracking the index fingertip, placing the tracking element on the back of the hand might cause additional errors. Moreover, also the calibration superimposing the virtual and real fingertips could led to mismatches between the virtual model and the real hand. Our approach aims at mitigating these different types of errors by providing a direct measure of the distance with respect to the tangible object.

The "touching" and "tapping" tasks were performed on the three instrumented faces of the cube: top, back, and right side with respect to the user (see Fig. 2), so as to test different visibility conditions of the user on the contact area. The cube was positioned slightly on the left of the user so that he or she clearly saw the right face from the side. Moreover, it was placed close to the table edge so as to enable the user to easily reach its hidden face on the back. Participants always wore both tracking elements (i.e., the HTC Vive tracker and the Vicon's reflective markers) to ensure a consistent calibration throughout the experiment and avoid suggesting to the user which tracking system was in use.

The experiment was split in 6 blocks, so as to have all combinations between the two tracking systems and the three faces of the cube. The ordering of the three faces was counterbalanced following a Latin square. Participants did the two blocks with the two tracking systems in a row for each cube face. The order between the two tracking systems was counter-balanced for each cube face. In each block, the participants always did first the "touching" task then the "tapping" task. And for each task, there were 6 repetitions, with and without capacitive sensing correction for a total of 12 trials per task presented in a randomized order. Thus, each participant did 72 trials per task, for a total number of 144 trials for the whole experiment, which lasted an hour.

\subsubsection{Experimental variables}

We considered four experimental conditions:
C1: Capacitive sensing (E: Enabled, D: Disabled)
C2: Optical tracking (T: Vive Tracker, V: Vicon Bonita)
C3: Cube face (Tf: Top, Sf: Side, Bf: Back)
C4: Task (Touching, Tapping)

We considered all combinations of these four experimental conditions, yielding 24 experimental modalities.

\subsubsection{Collected Data}

For each trial, we collected the participants' answers regarding the coherence of the contact (CC) on a 7-item Likert scale (Q0 in Sec. 4.1.3), as well as, for each frame, the optical tracking data, the corrected position as per our approach, the virtual distance between the virtual hand and the target face projected onto the face's normal, the capacitive sensing output, and whether the contact has happened. Then, we computed the mean error at contact (EC) for each trial, i.e., the mean virtual gap or interpenetration distance displayed to the participant whenever the physical/tangible contact occurred. At the end of the experiment, participants filled a subjective questionnaire using a 7-item Likert scale. The questions were (Q1): It felt like tapping a real cube; (Q2): It felt like touching a real cube; (Q3): It felt like I was seeing my own hand; (Q4): I felt tired at the end.

\subsection{Experimental results}

Contact coherency (CC). To study the participants answers on contact coherency $(\mathrm{Q} 0)$, we used a linear mixed model for the four conditions $\mathbf{C} 1, \mathbf{C} 2, \mathbf{C} 3$ and $\mathbf{C} 4$. We performed a stepwise selection of the model variables. The participants were considered as a random effect in the model. Our analysis of variance on the selected model showed a significant effect of C1, C2, C3 $(F(1,2560)=792.55, F(1,2560)=626.37$ and $F(2,2560)=106.42$ respectively, $p<0.001$ for the three conditions). No significant effect was found for C4, the task condition $(p=0.91)$. The coherency ratings for both tasks were very similar $(M=4.5, S D=1.92$ for the static task, $M=4.51, S D=1.97$ for the dynamic task). Concerning C1, there was a significant difference in the ratings when the capacitive system was activated $(M=5.28$, $S D=1.44)$ or not $(M=3.72, S D=2.06)$. Concerning C2, there was a significant difference in the ratings between the two tracking systems $(M=5.2, S D=1.63$ for the Vicon system, $M=3.81, S D=1.98$ for the HTC Vive system). Finally, there were significant differences between the three faces of the cube $(M=4.89, S D=1.84$ for the top face, $M=3.95, S D=1.99$ for the side face, $M=4.67$, $S D=1.86$ for the back face). Results are summarized in Table 1. An interaction effect was found between $\mathbf{C} \mathbf{1}$ and $\mathbf{C 2}$ $(p<0.001)$. A $C h i^{2}$ test showed that whatever the capacitive system was enabled or not, there was a significant different between the two tracking systems ( $p<0.001$ for both tests). Similarly whatever the tracking system used, there was a significant difference between the two states of activation 
of the capacitive sensing ( $p<0.001$ for both tests). An interaction effect was also found between C1 and C3 $(p<$ $0.001)$. We also found a significant effect between $\mathbf{C} 2$ and C3 $(p<0.001)$, and between $\mathbf{C} 3$ and $\mathbf{C 4}(p<0.001)$.

TABLE 1

Contact Coherency (CC) scores and Error at Contact (EC) in millimeters. Mean (Standard Deviation) for the two optical tracking systems and the three faces of the cube, depending on the state of the capacitive system (D: Disabled, E: Enabled).

\begin{tabular}{|c|c|c|c|c|}
\cline { 3 - 5 } \multicolumn{1}{c|}{} & Top face (Tf) & Side face (Sf) & Back Face (Bf) \\
\hline \multirow{4}{*}{ CC } & \multirow{3}{*}{ Vicon (V) } & D: $5.60(1.32)$ & D: $2.75(1.31)$ & D: $5.57(1.24)$ \\
& & E: $6.06(0.93)$ & E: $5.61(1.08)$ & E: $5.58(1.25)$ \\
\cline { 2 - 5 } & \multirow{3}{*}{ Vive (T) } & D: $2.87(1.78)$ & D: $2.68(1.97)$ & D: $2.89(1.77)$ \\
& & E: $5.05(1.33)$ & E: $4.76(1.64)$ & E: $4.63(1.68)$ \\
\hline \multirow{3}{*}{ EC } & \multirow{2}{*}{ Vicon (V) } & D: $2.75(1.92)$ & D: $7.94(2.07)$ & D: $3.06(2.59)$ \\
& \multirow{2}{*}{ Vive (T) } & E: $0.18(0.20)$ & E: $0.15(0.16)$ & E: $0.16(0.17)$ \\
\cline { 3 - 5 } & & D: $14.9(14.8)$ & D: $-8.38(17.5)$ & D: $27.8(13.2)$ \\
& E: $0.17(0.18)$ & E: $0.16(0.17)$ & E: $0.15(0.17)$ \\
\hline
\end{tabular}
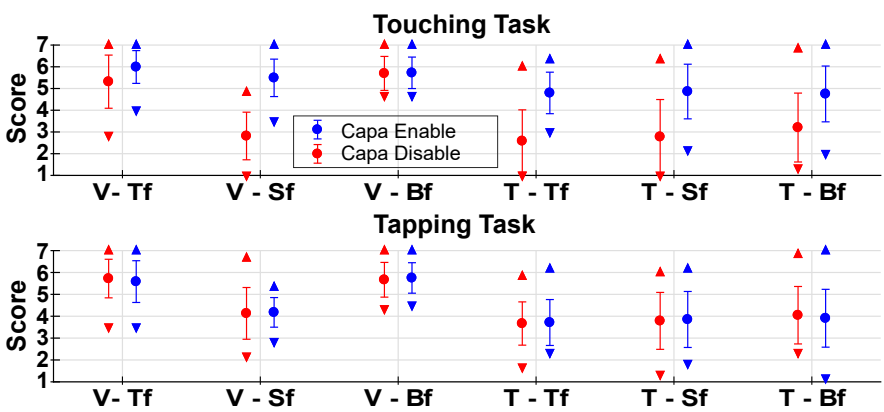

Fig. 5. Contact coherency (CC) score, i.e., answer to Q0. Score over all experimental modalities, for both optical tracking systems $(T, V)$, with or without capacitive correction (E, D), cube face (Tf, Sf, Bf), and the two tasks (Touching, Tapping). The mean, standard deviation, min, and max values are represented by dots, bars, and triangles, respectively.
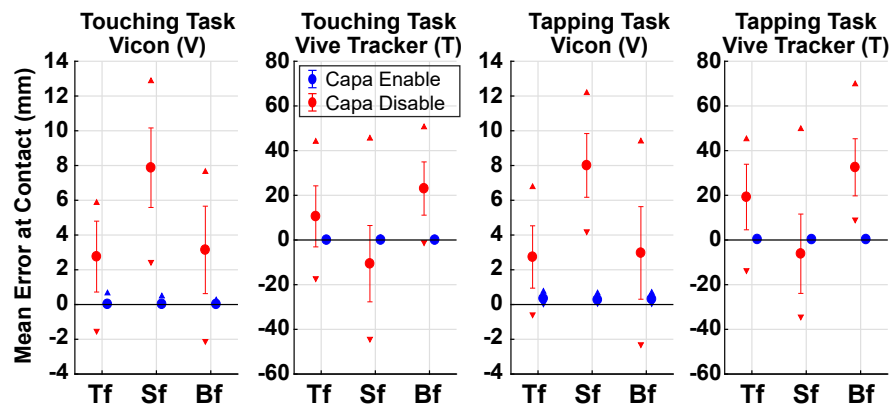

Fig. 6. Error at Contact (EC) in mm over all experimental modalities.

Error at Contact (EC). To study the mean error at contact, we used a linear mixed model on the collected data for the four conditions $\mathbf{C} \mathbf{1}, \mathbf{C} 2, \mathbf{C} 3$, and $\mathbf{C 4}$. We performed a stepwise selection of the model variables. The participants were considered as a random effect in the model. Our analysis of variance on the selected model showed a significant effect of C1, C2, and C3 $(F(1,2547)=485.61, F(1,2547)=160.75$ and $F(2,2547)=74.53$ respectively, $p<0.001$ for all the conditions). An interaction effect was found between C2 and all the other conditions $(p<0.001)$. We also found an interaction effect between C1 and C3 $(p<0.001)$.

Subjective questionnaire. The subjective questionnaire (Fig. 7) shows that, overall, participants felt like tapping and touching a real object, although scores are a bit higher for the latter. Participants also rather agree that the virtual hand felt like their own, although this result falls into the mid range of the scores.

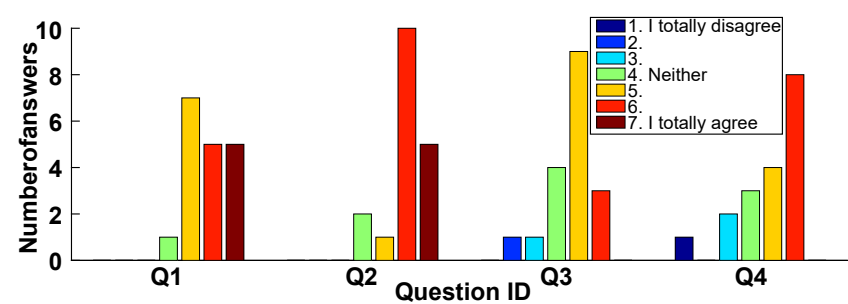

Fig. 7. Bar-graph of answers for the subjective questions. Q1: It felt like tapping real cube (M: 5.78 SD: 0.94 ), Q2: It felt like touching real cube (M: 6 SD: 0.91), Q3: It felt like I was seeing my own hand (M: 4.67 SD 1.03), Q4: I felt tired at the end (M: 4.83 SD: 1.42$)$

\section{Discussion}

As the statistical analysis shows a significant effect on condition $\mathrm{C} 1$ and the contact coherence (CC) ratings are higher whenever the capacitive sensing is enabled (see Fig. 5 and Table 1), we can conclude that our capacitive-sensingbased approach does increase the overall contact rendering coherency. Additionally, the interaction effect between C1 and $\mathbf{C} 2$ shows that for both tracking systems independently, the difference is significant regardless of the activation of the capacitive sensing. This significant difference corresponds to an increase of the rating when capacitive sensing is enabled. Thus, $\mathrm{H} 1$ is validated.

On the other hand, $\mathrm{H} 2$ is not validated, as no significant effect was found on $\mathbf{C 4}$. The mean increase of rating for the touching task is clearly visible in Fig. 5. At least, the capacitive sensing did not decrease the ratings, suggesting that it can only improve the perceived coherency from brief to maintained contacts.

Interacting with the side face changes the behavior of the participants, as the statistical analysis showed a significant effect on C3. Indeed, as subjects had a clear line of sight of the side face, they would notice more any inconsistency at the contact. This can be observed when the Vicon is used (Fig. 5). In this case, the ratings are lower than for other faces because of the remaining visible gap when the capacitive sensing is not used. As expected, adding the capacitive sensing increased the rating because of the reduction of the gap. But this behavior is not observed when using the Vive Tracker (Fig. 5). Thus, H3 is not validated.

We also used a metric to monitor the remaining error at contact (Table 1, EC). When adding the capacitive sensing, the error at contact is brought down below the millimeter's level in all conditions, supporting hypotheses $\mathrm{H} 1$ and $\mathrm{H} 2$. This estimation still shows a remaining error up to $0.5 \mathrm{~mm}$ in some cases, as the sensor's threshold for breaking contact is higher than for making contact, thus leading to a slight overestimation of the distance at contact, especially for the tapping task. Fig. 6 shows that the remaining error when using the Vicon is two times higher for the side face than for the two others when capacitive sensing is disabled. The fact that this error is higher even when using the Vicon suggests a setup calibration error, which might also be one of the reasons why the scoring is lower on this face compared to the others, thus hindering hypothesis H3. As shown in Fig. 6 and Table 1, using the Vicon led to a constant gap/error, while using the Vive Tracker led to much more variation, 
with cases of visual interpenetration upon contact. This issue might have affected the participant's behavior and be an additional reason why $\mathrm{H} 3$ could not be validated.

Regardless of the considered condition, whenever the capacitive sensing was used, the error at contact was drastically reduced, i.e., calibration errors and mismatches were mitigated. In light of these results, the proposed approach of combining capacitive sensing on the tangible object with external optical tracking seems an effective way to improve visuohaptic coherency of contact in VR, even if the tracking system used is not the most accurate. The evaluation of the mean and standard deviation of the error at contact (without capacitive correction, in red on Fig. 6) suggests that using the Vicon markers on the distal phalanx is indeed a more precise and accurate solution than using a Vive Tracker on the back of the hand. Indeed, the former has a remaining error in the order of millimeters while the latter in that of centimeters. Even when the capacitive sensing is enabled, there is still a significant difference in the ratings between the two tracking systems. In this respect, our capacitive sensing approach can make an affordable tracking system such as the Vive Tracker a viable and good solution for interacting in VR. Indeed, the addition of capacitive sensing to the Vive Tracker improves the tracking coherency, provides a more homogeneous behavior between faces, and reduce the overall error at contact. Furthermore, capacitive sensing can also provide absolute contact information, i.e., a direct information about whether the contact has happened. This feature can be used as an event trigger, instead of, e.g., using often unreliable position-based estimations of contact from an external optical tracking system. An example is using capacitive sensing on an tangible object to detect when the user grasps/releases it.

Although we only instrumented the flat surface of a cube, it is possible to instrument surfaces of any curvature. In fact, only the proximity of the user from the conductive electrode influences the circuit capacity $C_{p}$ (see Sec. 3.2). Additionally, one can also change the sensibility of the oscillator, i.e., the detection range, by changing $C_{0}$ : the smaller $C_{0}$ is, the smaller variations of $C_{p}$ can be detected, the larger the detection range is. In addition, the size of the electrode also impacts the range of detection and the width of the detection area. Therefore, the circuit elements should be adjusted according to the considered application. Finally, our approach is compatible and complementary with prior studies [4], [6], [7], [29], leading toward generic tabletop VR setups using a small number of dedicated tangible objects compatible with a wide variety of virtual scenarios.

The proposed approach is rather robust to changes in hand pose/orientation. As all parts of the hand contribute to the capacitive readings, if the pose differs significantly from what we expected, the user might experience some accelerated/slowed down motions of the virtual hand with respect to the real one, which will be mitigated as the user moves toward the instrumented object. However, as the fingertip approaches the sensor whilst being the closest part to the sensor, its contribution to the sensed capacity increases significantly. This makes the other parts of the hand less and less important, making the estimated position converge toward the fitted behavior. Another limitation is that a calibration is needed to determine the resting resonance frequency $f_{0}$ of the circuitry when a new electrode is plugged.
In addition, this frequency tends to shift with temperature. This issue can be addressed by implementing an algorithm regularly checking the value of $f_{0}$ whenever the user is outside the capacitive detection range. Lastly, our capacitive system needs to be connected to the detection circuit, making it difficult to move the tangible object away from its resting position. A solution could be to design a docking station where the tangible object is automatically connected to the detection circuitry when docked.

\section{Conclusion And Future Work}

We presented a new approach for using tangible objects in VR, so as to achieve high-quality contact rendering through visuohaptic contact synchronicity between the tangible and virtual environments. By instrumenting a tangible object with capacitive sensing, we could reliably estimate the user's proximity to this object. Then, we proposed a visuohaptic retargeting technique to combine the capacitive sensing information with that of an external optical tracking framework. Seamlessly merging these two pieces of information enables to adjust the position of the user's virtual finger with respect to the tangible object along the normal at its surface. This approach mitigates any relative positioning errors and avoid interpenetrations or gaps between the virtual object and the hand avatar whenever the user's hand is in contact with the corresponding tangible object.

We tested our approach in a user study enrolling 18 participants. They were asked to interact with a tangible cube in immersive VR and assess the coherency of the virtual and tangible contacts, either using only an external optical tracking (standard approach) or combining optical tracking with capacitive sensing (our approach). We considered two optical tracking systems (HTC Vive; Vicon Bonita) and two interaction tasks (tapping on the object; approaching and touching it), yielding a total of 144 interactions per participant. Results show that our approach significantly increased the perceived coherency and synchronicity of the VR experience, correcting common relative positioning errors related to the use of optical tracking systems and tangible objects. The proposed approach is compatible with any type of external tracking system and can be easily extended to different types of virtual environments and tangible objects.

In the future, we will work on better characterizing the relationship between the sensed capacity and the user's proximity, considering machine learning approaches and a larger data set. We will also analyze more in depth the relationship between the user's hand pose and the quality of the interaction, so as to evaluate the sensitivity of the system to this variable. Finally, we will study how to extend the sensing range of the system by combining multiple electrodes as well as analyze how to best treat sudden changes in the capacity (e.g., if the hand enters the electrode sensing range from the side).

\section{REFERENCES}

[1] M. Di Luca and A. Mahnan, "Perceptual limits of visual-haptic simultaneity in virtual reality interactions," in Proc. IEEE World Haptics Conference, 2019, pp. 67-72.

[2] M. Di Luca, B. Knörlein, M. O. Ernst, and M. Harders, "Effects of visual-haptic asynchronies and loading-unloading movements on compliance perception," Brain research bulletin, vol. 85, no. 5, pp. 245-259, 2011. 
[3] B. Knorlein, M. Di Luca, and M. Harders, "Influence of visual and haptic delays on stiffness perception in augmented reality," in Proc. IEEE International Symp. Mixed and Augmented Reality, 2009, pp. 49-52.

[4] J. Arora, A. Saini, N. Mehra, V. Jain, S. Shrey, and A. Parnami, "Virtualbricks: Exploring a scalable, modular toolkit for enabling physical manipulation in vr," in Proc. CHI Conf. Human Factors in Computing Systems, 2019, pp. 1-12.

[5] S. V. Salazar, C. Pacchierotti, X. de Tinguy, A. Maciel, and M. Marchal, "Altering the stiffness, friction, and shape perception of tangible objects in virtual reality using wearable haptics," IEEE Trans. Haptics, vol. 13, no. 1, pp. 167-174, 2020.

[6] D. T. Han, M. Suhail, and E. D. Ragan, "Evaluating remapped physical reach for hand interactions with passive haptics in virtual reality," IEEE Trans. Visualization and Computer Graphics, vol. 24, no. 4, pp. 1467-1476, 2018.

[7] X. de Tinguy, C. Pacchierotti, M. Marchal, and A. Lécuyer, "Toward universal tangible objects: Optimizing haptic pinching sensations in 3d interaction," in IEEE Conf. Virtual Reality and 3D User Interfaces, 2019.

[8] X. de Tinguy, C. Pacchierotti, M. Emily, M. Chevalier, A. Guignardat, M. Guillaudeux, C. Six, A. Lécuyer, and M. Marchal, "How different tangible and virtual objects can be while still feeling the same?" in Proc. IEEE World Haptics Conference, 2019, pp. 580-585.

[9] K. Hinckley and M. Sinclair, "Touch-sensing input devices," in Proc. CHI Conf. Human Factors in Computing Systems, 1999, pp. 223-230.

[10] M. Sato, I. Poupyrev, and C. Harrison, "Touché: enhancing touch interaction on humans, screens, liquids, and everyday objects," in Proc. CHI Conf. Human Factors in Computing Systems, 2012, pp. 483-492.

[11] G. Barrett and R. Omote, "Projected-capacitive touch technology," Information Display, vol. 26, no. 3, pp. 16-21, 2010.

[12] N.-H. Yu, L.-W. Chan, S. Y. Lau, S.-S. Tsai, I.-C. Hsiao, D.-J. Tsai, F.-I. Hsiao, L.-P. Cheng, M. Chen, P. Huang, et al., "Tuic: enabling tangible interaction on capacitive multi-touch displays," in Proc. CHI Conf. Human Factors in Computing Systems, 2011, pp. 2995-3004

[13] J. Rekimoto, "Smartskin: an infrastructure for freehand manipulation on interactive surfaces," in Proc. CHI Conf. Human factors in computing systems, 2002, pp. 113-120.

[14] S. Phan, Z. F. Quek, P. Shah, D. Shin, Z. Ahmed, O. Khatib, and M. Cutkosky, "Capacitive skin sensors for robot impact monitoring," in Proc. IEEE/RSJ International Conf. Intelligent Robots and Systems, 2011, pp. 2992-2997.

[15] C. Bartolozzi, P. M. Ros, F. Diotalevi, N. Jamali, L. Natale, M. Crepaldi, and D. Demarchi, "Event-driven encoding of offthe-shelf tactile sensors for compression and latency optimisation for robotic skin," in Proc. IEEE/RSJ International Conf. Intelligent Robots and Systems, 2017, pp. 166-173.

[16] J. MacRitchie and A. P. McPherson, "Integrating optical finger motion tracking with surface touch events," Frontiers in Psychology, vol. 6, p. 702, 2015.

[17] F. Chinello, C. Pacchierotti, M. Malvezzi, and D. Prattichizzo, "A three revolute-revolute-spherical wearable fingertip cutaneous device for stiffness rendering," IEEE Trans. Haptics, vol. 11, no. 1, pp. 39-50, 2017.

[18] F. Chinello, M. Malvezzi, D. Prattichizzo, and C. Pacchierotti, “A modular wearable finger interface for cutaneous and kinesthetic interaction: control and evaluation," IEEE Trans. Industrial Electronics, vol. 67 , no. 1, pp. 706-716, 2019.

[19] V. Vechev, J. Zarate, D. Lindlbauer, R. Hinchet, H. Shea, and O. Hilliges, "Tactiles: Dual-mode low-power electromagnetic actuators for rendering continuous contact and spatial haptic patterns in vr," in Proc. IEEE Conf. Virtual Reality and 3D User Interfaces, 2019, pp. 312-320.

[20] K. J. Kuchenbecker, D. Ferguson, M. Kutzer, M. Moses, and A. M. Okamura, "The touch thimble: Providing fingertip contact feedback during point-force haptic interaction," in Proc. IEEE Symposium on Haptic Interfaces for Virtual Environment and Teleoperator Systems, 2008, pp. 239-246.

[21] E. M. Young, D. Gueorguiev, K. J. Kuchenbecker, and C. Pacchierotti, "Compensating for fingertip size to render tactile cues more accurately," IEEE Trans. Haptics, vol. 13, no. 1, pp. 144-151, 2020.

[22] L. Kohli, "Exploiting perceptual illusions to enhance passive haptics," in Proc. IEEE VR Workshop on Perceptual Illusions in Virtual Environments, 2009, pp. 22-24.

[23] J. Sreng, A. Lécuyer, C. Mégard, and C. Andriot, “Using visual cues of contact to improve interactive manipulation of virtual objects in industrial assembly/maintenance simulations," IEEE
Trans. Visualization and Computer Graphics, vol. 12, no. 5, pp. 10131020,2006

[24] L.-P. Cheng, E. Ofek, C. Holz, H. Benko, and A. D. Wilson, "Sparse haptic proxy: Touch feedback in virtual environments using a general passive prop," in Proc. CHI Conf. Human Factors in Computing Systems, 2017, pp. 3718-3728.

[25] L. Meli, C. Pacchierotti, G. Salvietti, F. Chinello, M. Maisto, A. De Luca, and D. Prattichizzo, "Combining wearable finger haptics and augmented reality: User evaluation using an external camera and the microsoft hololens," IEEE Robotics and Automation Letters, vol. 3, no. 4, pp. 4297-4304, 2018.

[26] J. Bimbo, C. Pacchierotti, M. Aggravi, N. Tsagarakis, and D. Prattichizzo, "Teleoperation in cluttered environments using wearable haptic feedback," in IEEE/RSJ International Conf. Intelligent Robots and Systems, 2017, pp. 3401-3408.

[27] T. Lisini Baldi et al., "GESTO: A glove for enhanced sensing and touching based on inertial and magnetic sensors for hand tracking and cutaneous feedback," IEEE Trans. Human-Machine Systems, vol. 47, no. 6, pp. 1066-1076, 2017.

[28] J. McIntosh, P. Strohmeier, J. Knibbe, S. Boring, and K. Hornbæk, "Magnetips: Combining fingertip tracking and haptic feedback for around-device interaction," in Proc. CHI Conf. Human Factors in Computing Systems, 2019.

[29] M. Azmandian, M. Hancock, H. Benko, E. Ofek, and A. D. Wilson, "Haptic retargeting: Dynamic repurposing of passive haptics for enhanced virtual reality experiences," in Proc. CHI Conf. Human Factors in Computing Systems, 2016, pp. 1968-1979.

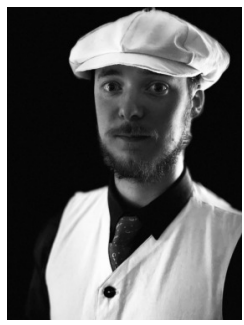

Xavier de Tinguy received a Master Degree in Embedded System and Information Processing from l'Université Paris-Sud and graduated from Ecole Normale Supérieur de Paris-Saclay in Electrical Engineering in 2017. He is a doctoral student working in the Hybrid research team in Inria Rennes, France. His main research interests include virtual reality, interaction techniques wearable haptic interface, and tangibility within VR.

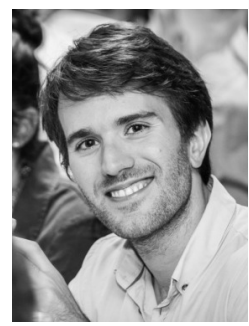

Claudio Pacchierotti is a researcher of the CNRS at IRISA and Inria Rennes, France. He earned his $\mathrm{PhD}$ degree at the University of Siena in 2014, and he was then a postdoctoral researcher at the Italian Institute of Technology, Genova, Italy. Pacchierotti received the 2014 EuroHaptics Best PhD Thesis Award for the best doctoral thesis in the field of haptics. He is Chair of the IEEE Technical Committee on Haptics and Secretary of the Eurohaptics Society.

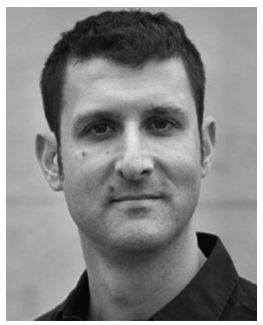

Anatole Lécuyer is a senior researcher and head of Hybrid team, Inria Rennes, France. He is currently an associate editor of the IEEE Transactions on Visualization and Computer Graphics, Frontiers in Virtual Environments, and Presence journals, as well as formerly of the ACM Transactions on Applied Perception and the International Journal of Humanities and Cultural Studies. He was a program chair of the IEEE Virtual Reality Conference (2015-2016) and the IEEE Symposium on 3D User Interfaces (2012-2013).

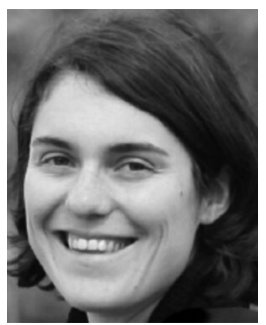

Maud Marchal received the $\mathrm{PhD}$ degree in computer science from the University Joseph Fourier, in Grenoble, France, in 2006. She is an associate professor with the Computer Science Department, INSA Rennes, France. She is an associate editor of the IEEE Transactions on Visualization and Computer Graphics. She is currently program chair of the IEEE Virtual Reality Conference Journal Track (2020-2021) and was program chair of the Conference Track (2018) and the IEEE Symposium on 3D User Interfaces (2016-2017). 